\title{
Viewpoint Development of Stochastic Hybrid Systems
}

\author{
Manuela L. Bujorianu and Marius C. Bujorianu
}

\begin{abstract}
Nowadays, due to the explosive spreading of networked and highly distributed systems, mastering system complexity becomes a critical issue. Two development and verification paradigms have become more popular: viewpoints and randomisation. The viewpoints offer large freedom and introduce concurrency and compositionality in the development process. Randomisation is now a traditional method for reducing complexity (comparing with deterministic models) and it offers finer analytical analysis tools (quantification over nondeterminism, multi-valued logics, etc). In this paper, we propose a combination of these two paradigms introducing a viewpoint methodology for systems with stochastic behaviours.
\end{abstract}

Keywords: stochastic hybrid systems, Markov processes, viewpoints, formal development, simulation, probabilistic kernels.

\section{INTRODUCTION}

Viewpoints, or multi-perspectives, constitute now a mature development paradigm summing a large palette of methodologies, results and applications. This paradigm has observed more than fifteen years of research and more than one hundred publications. Viewpoints have been standardized now, for example by ISO in the Reference Model for Open Distributed Processing, RM-ODP, standard [22] and by IEEE in the P1471 standard [21]. The RM-ODP viewpoint model has been extended [17] by European Space Agency (ESA) to use UML/XML open standard technologies for space system and information modelling to improve data exchange. They originate from networked and distributed systems, but also can be used for general software systems.

The modular development is the most obvious advantage of viewpoints. The major principle of viewpoints consists in the freedom offered to the viewpoint owners to choose their favorite computational model, specification language, refinement techniques, verification tool, etc. The simultaneous construction of the viewpoints implies further advantages as concurrent system verification, geographical distribution, formal support for heterogeneity, combination of tools, etc. However, the significant advantages pay back in the difficulty of problems associated with viewpoints: integration and consistency checking. Offering a particular perspective on the overall system, the viewpoints can have overlappings that can cause inconsistency and also they need to be integrated. The viewpoint methodologies start diverging from the way they approach these problems.

This work was supported by NWO [26]

Manuela L. Bujorianu is with Faculty of Computer Science, Unversity of Twente, The Netherlands L.M.Bujorianu@cs.utwente.nl

Marius C. Bujorianu is with Computer Laboratory, University of Kent, UK M.C.Bujorianu@kent.ac.uk
Probabilistic modelling, specification and verification [15], [7], know a wide spread and an explosive growth. Although, randomisation is thought of as an efficient method to reduce the complexity of deterministic systems, it is now the case that the probabilistic systems are getting very complex. Therefore, it is natural to consider viewpoint development of probabilistic systems. From a methodological perspective, such approach would be a powerful combination of a modelling technique with a system development one.

The potential applications of our methodology are various since we have used very general models. The fact that it applies also to continuous systems favors applications in fields like control engineering, air traffic control, etc. However, the large network systems like the Internet are now approximated by continuous systems [23], [24], the so-called fluid models. In computer networks, there are continuous models with well developed theory and tools like the generalized Fluid Stochastic Petri Nets models [29], [25]. The fluid models constitute an ideal application domain for any stochastic viewpoint development methodology.

The paper is structured as follows. In the next section we give a short background. Then, in Section 3, we define the stochastic viewpoints. In Section 4, we present the mathematical principles underpinning the viewpoint development methodology and we show how the integration process can be effectively constructed and we prove that this is a common simulation of the viewpoint processes. In the last section, we draw some partial conclusions and sketch some further work.

\section{Stochastic Viewpoints}

\section{A. A short primer on viewpoints}

A rich approach based on the classical foundations of specification and refinement has been developed by Derrick, Boiten, Bowman, e.a., known as the Kent group [6], [28]. In their approach, viewpoint specifications are linked by relations, called correspondences, and consistency is checked for the connected identifiers. The existence of a sound, common refinement means viewpoint consistency. In the refinement ordering, of a special interest are the minimal integrations, called unifications. A unification method has been constructed in the relational framework underlying the object- $Z$ specification language. Heterogeneity is treated by translating all viewpoints into the relational framework. In some recent developments, the relational approach has been generalised categorically to a framework called correspondence carrying specifications [11], [12].

However, the principles of this approach might be too strong in the new technological context. Nowadays, the 
system architects move their interest from a minimal integration to more flexible composition operators that preserve, for example, performability and QoS requirements. Rich and expressive correspondences imply laborious consistency check and therefore some system engineers prefer viewpoint decomposition with limited interference (or rather components with clever interfaces).

The other viewpoint methodologies adopt a more tolerant view and are based on non-classical logics. Finkelstein e.a. [19] have constructed a rich engineered framework, called Viewpoints, in which inconsistency is tolerated. Their philosophy is that the main source of inconsistency is due to incomplete information in the early development stages and it will be removed in latter stages.

In this paper, we develop a viewpoint methodology, in the spirit of correspondence carrying specifications, by considering a relaxed form of integration. The main idea is to renounce at the determinism conditions and consider viewpoints that are probabilistic systems. The correspondence relations are rather unbalanced, in the sense that two correspondent objects are not considered semantically equal or bisimilar, but one being a refinement of another. The unification mirrors the pushout construction by simulating all viewpoints and eliminating duplicate components (i.e., it considers only one of two correspondent components). The refinement relation we consider is given by zigzag morphisms.

\section{B. Viewpoint definition}

A stochastic process $M$ is called a Borel right process with the state space $(X, \mathcal{B})$ if the following conditions are fulfilled.

1. $M$ is a strong Markov process with right-continuous paths. 2. $X$ is a separable metric space homeomorphic to a Borel subset of some compact metric space, equipped with Borel $\sigma$-algebra $\mathcal{B}(X)$ or shortly $\mathcal{B}$. This means $X$ is a Lusin state space.

3. The operator semigroup of $M$, given by

$$
P_{t} f(x)=E_{x} f\left(x_{t}\right)
$$

where $E_{x}$ is the expectation w.r.t. $P_{x}$ maps $\mathcal{B}^{b}(X)$ (the lattice of bounded real measurable functions defined on $X)^{1}$ into itself.

4. If $f$ is an $\alpha$-excessive function for $\mathcal{P}$, then the sample path $t \rightarrow f\left(x_{t}(\omega)\right)$ is a.s. right continuous. Recall that a nonnegative function $f \in \mathcal{B}^{b}(X)$ is called $\alpha$-excessive $(\alpha>$ $0)$ if $e^{-\alpha t} P_{t} f \leq f$ for all $t \geq 0$ and $e^{-\alpha t} P_{t} f \nearrow f$ as $t \searrow 0$.

Suppose we have given $n \in \mathbb{N}_{(}(n \geq 2)$ Borel right processes $\widehat{M}_{i}$ with the state spaces $\widehat{X}_{i}$, for $i=1, . . n$. Each space $\widehat{X}_{i}$ comes with its Borel $\sigma$-algebra $\mathcal{B}\left(\widehat{X}_{i}\right)$. In our terminology $\widehat{M}_{i}$ will be called viewpoint processes.

Let us consider a Borel right process $M$ with the state space $(X, \mathcal{B}(X))$. Moreover, we assume that the process $M$ is 'simulated' by the viewpoint processes locally. Then, the

\footnotetext{
${ }^{1}$ Usually, in this paper $\mathcal{B}^{b}(S)$ is the lattice of bounded real measurable functions defined on a topologic space $S$.
}

research problem, which derives from here is to integrate the viewpoint processes in order to obtain a global process that simulates the whole process $M$.

Formally, assume there exist a partition of the state space $X$ with the closed sets

$$
X=\cup_{i=1}^{n} A_{i}, A_{i} \cap A_{j}=\emptyset \text { if } i \neq j,
$$

and $n$ surjective continuous maps $\psi_{i}: X \rightarrow \widehat{X}_{i}, i=1, . ., n$ such that $\psi_{i}^{-1}\left(\psi_{i}\left(A_{i}\right)\right)=A_{i}$. The sets $A_{i}$ can be thought of as the closures of the modes of the stochastic system $H$. The maps $\psi_{i}$ satisfy the zigzag morphism condition in the sense of [10], i.e. the processes $\widehat{M}_{i}$ simulate the process $M$ on the set $\psi_{i}\left(A_{i}\right), i=1, . ., n$.

We briefly remind the concept of zigzag morphism [10], which will be used in this paper. A surjective continuous mapping $\psi: X^{\prime} \rightarrow X$ between the state spaces of two processes $M^{\prime}$ and $M$ is called zigzag morphism if the following condition holds

$$
P_{t}^{\prime}(f \circ \psi)=P_{t} f \circ \psi, \forall f \in \mathcal{B}^{b}(X), t>0
$$

where $\left(P_{t}^{\prime}\right)$ (respectiv $\left(P_{t}\right)$ ) is the semigroup of $M^{\prime}$ (respectiv $M$ ). In this case, we say that $M$ simulates $M^{\prime}$. Intuitively, the zigzag morphism condition states that the transition probabilities of the process $M^{\prime}$ corresponding to the sets closed under the relation generated by $\psi$ are equal with the transition probabilities of $M$.

Therefore, in this work, we approach the problem how to construct an integration process $\overline{\mathrm{M}}$ defined on

$$
\widetilde{\mathbf{X}}=\cup_{i=1}^{n} \psi_{i}\left(A_{i}\right)
$$

which is still a Markov process and behaves as $\widehat{M}_{i}$ on $\psi_{i}\left(A_{i}\right), i=1, \ldots, n$. This process will represent a simulating process of $M$.

\section{Methodology Principles}

Let $X$ and $\widehat{X}$ be two Polish spaces (i.e. the topology of $X$ or $\widehat{X}$ is complete, separable, and metrizable). Let

$$
\psi: X \rightarrow \widehat{X}
$$

be a continuous surjection. Then the topological space is decomposed in two components: a closed set $A \subseteq X$ and its complement $X \backslash A$. Then we define

$$
\widetilde{X}:=(X \backslash A) \cup \psi(A),
$$

this being a disjoint union. We assume also that

$$
\psi^{-1}(\psi(A))=A
$$

Note that we are not assuming that $\psi$ is one to one. Condition (4) ensures that $\psi$ can be restricted as a surjective map defined on $A$ with values in $\psi(A)$.

Suppose that we have a nice Markov process $M$ with the state space $X$. In [18], it is presented a construction of a Markov process $\widetilde{M}$ on $\widetilde{X}$ by pinching $M$ to $\psi \circ M$ when $M$ is in $A$, but keeping the initial dynamics of $M$ when it is in $X \backslash A$. The cornerstone of this construction is what happens when $\widetilde{M}$ leaves $X \backslash A$ and enters $\psi(A)$ or viceversa. 
Let us define the "pinching" map $\pi: X \rightarrow \widetilde{X}$ by

$$
\pi(x)= \begin{cases}x & \text { if } x \in X \backslash A \\ \psi(x) & \text { if } x \in A .\end{cases}
$$

The pinching map $\pi$ is injective on $X \backslash A$, i.e. no pinching occurs on $X \backslash A$, but is not generally injective on $A$. The space $\widetilde{X}$ will be equipped with the topology induced by $\pi$, i.e. $G \subseteq \widetilde{X}$ is open in the topology of $\widetilde{X}$ if and only if $\pi^{-1}(G)$ is open in the topology of $X$. The space $\widetilde{X}$ can be thought of as the quotient topological space $X$ under the following equivalence relation

$$
x \mathcal{R} y \Leftrightarrow \pi(x)=\pi(y)
$$

We assume that $\widetilde{X}$ with this topology is a Polish space. The topology of $\widetilde{X}$ is equivalent to that of $X$ on $X \backslash A$ and to that of $\widehat{X}$ on $\psi(A)$, but the points on the boundary of $X \backslash A$ (in the topology of $X$ ) are identified with the points on the boundary of $\psi(A)$ (in the topology of $\widehat{X}$ ) via the map $\psi$ [18].

The spaces $X, \widetilde{X}$ and $\widehat{X}$ will be endowed with their Borel $\sigma$-algebras $\mathcal{B}(X), \mathcal{B}(\widetilde{X})$, and, respectively, $\mathcal{B}(\widehat{X})$. by

There exists a natural continuous map $\phi: \widetilde{X} \rightarrow \widehat{X}$ defined

$$
\phi(x)= \begin{cases}\psi(\widetilde{x}) & \text { if } \widetilde{x} \in X \backslash A \\ \widetilde{x} & \text { if } \widetilde{x} \in \psi(A) .\end{cases}
$$

Obviously, $\psi=\phi \circ \pi$ or the following diagram commutes

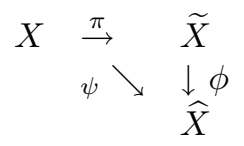

Let us consider the lattices $\mathcal{B}^{b}(X), \mathcal{B}^{b}(\widetilde{X})$ and respectively $\mathcal{B}^{b}(\widehat{X})$ of bounded real-valued measurable defined on $X, \widetilde{X}$ and respectively $\widehat{X}$. Then, one might define the ${ }^{*}$-maps

$$
\begin{array}{ll}
\psi^{*} \quad: \quad \mathcal{B}^{b}(\widehat{X}) \rightarrow \mathcal{B}^{b}(X), \psi^{*} f=f \circ \psi \\
\pi^{*}: \quad \mathcal{B}^{b}(\widetilde{X}) \rightarrow \mathcal{B}^{b}(X), \pi^{*} f=f \circ \pi \\
\phi^{*}: \quad \mathcal{B}^{b}(\widehat{X}) \rightarrow \mathcal{B}^{b}(\widetilde{X}), \phi^{*} f=f \circ \phi
\end{array}
$$

such that $\pi^{*} \circ \phi^{*}=\psi^{*}$.

Remark 1: Condition (4) ensures that $\psi^{*}$ can be restricted to $\mathcal{B}^{b}\left(\psi(A)\right.$ ) with values in $\mathcal{B}^{b}(A)$ (where $\psi(A)$ and $A$ are equipped with the trace topologies).

Let us consider a Borel right process $M=$ $\left(\Omega, \mathcal{F}, \mathcal{F}_{t}, x_{t}, P^{x}\right)$ with the state space $X$ and the transition semigroup $\left(P_{t}\right)$. In order to obtain a new Markov process on $\widetilde{X}$, we have to impose the following assumption.

Assumption 1 (Dynkin intertwining relation): [16] We suppose that there exists a Borel right process denoted by $\widehat{M}=\left(\widehat{\Omega}, \widehat{\mathcal{F}}, \widehat{\mathcal{F}}_{t}, \widehat{x}_{t}, \widehat{P}^{x}\right)$ on the state space $\widehat{X}$ with the transition semigroup $\left(\widehat{P}_{t}\right)$ such that

$$
P_{t} \psi^{*}=\psi^{*} \widehat{P}_{t} .
$$

where $\psi^{*}$ is given by (6).

This implies that the finite dimensional distributions of $\psi \circ M$ under $P^{x}$ are the same as those of $\widehat{M}$ under $\widehat{P}^{\psi(x)}$ for any $x \in X$. The condition (9) says that $\psi$ is a Markov function, i.e. $\psi \circ M$ is still a Markov process [27].

Remark 2: The Assumption 1 and the conditions satisfied by $\psi$ ensure that $\psi$ is a zigzag morphism [10], i.e. $\widehat{M}$ simulates $M$.

Remark 3: In the light of the Remark 1, the Assumption 1 remains true for the semigroups of the 'killed' processes (i.e. the restriction of $M$ to $A$ and the restriction of $\widehat{M}$ to $\psi(A)$ ).

The goal is to obtain an $\widetilde{X}$-valued process $\widetilde{M}$, which behaves like $M$ when it is in $X \backslash A$ and like $\widehat{M}$ when it is in $\psi(A)$.

Ley $k: \widehat{X} \times \mathcal{B}(X) \rightarrow \mathbb{R}$ be a probability kernel, i.e. for each $\widehat{x} \in \widehat{X}, k(\widehat{x}, \cdot)$ is a probability measure on $(X, \mathcal{B}(X))$, and the map $\widehat{x} \mapsto k(\widehat{x}, B)$ is Borel measurable for each $B \in \mathcal{B}(X)$. This probability kernel is necessary in the construction of the desired process $\widetilde{M}$ to give the location where to jump in $X \backslash A$, if, for example, it starts on the boundary of $X \backslash A$ and $\psi(A)$ and decides to make an excursion in $X \backslash A$. Formally, if $\widetilde{M}$ would start in

$$
\widehat{x} \in \partial_{\widetilde{X}}(X \backslash A)=\partial_{\widetilde{X}} \psi(A),
$$

since $\psi^{-1}\{\widehat{x}\}$ might contain more than one point in $X$ and, in this case, $k(\widehat{x}, \cdot)$ would tell us where to jump in $X$.

Integrating w.r.t. $k(\widehat{x}, \cdot)$, one can define a linear operator $K$ : $\mathcal{B}^{b}(X) \rightarrow \mathcal{B}^{b}(\widehat{X})$ by

$$
(K f)(\widehat{x}):=\int f(y) k(\widehat{x}, d y) .
$$

Assumption 2: We assume that $k\left(\widehat{x}, \psi^{-1}(\widehat{x})\right)=1$, for all $\widehat{x} \in \widehat{X}$, i.e.

$$
k(\psi(x),[x])=1,
$$

for all $x \in X$ (here, $[x]$ is the equivalence of $x$ w.r.t. $\mathcal{R}$ defined by (5), $[x]$ is a measurable set of $X$ ).

Remark 4: Assumption 2 says that, for each $\widehat{x} \in \widehat{X}$ the probability measure $k(\widehat{x}, \cdot)$ is supported by $\psi^{-1}(\widehat{x})$. That means we can restrict the action of $K$ to $\mathcal{B}^{b}(A)$ having values in $\mathcal{B}^{b}(\psi(A))$.

The following assumption ensures that if $M$ has the initial probability distribution $k(\widehat{x}, \cdot)$, then $\psi \circ M$ is a Markov process with the initial state equal to $\widehat{x}$ [27].

Assumption 3: Assume that the semigroups $\left(P_{t}\right)$ and $\left(\widehat{P}_{t}\right)$ commute with $K$, i.e.

$$
K P_{t}=\widehat{P}_{t} K
$$

Note that the right hand side of (10) is

$$
\widehat{P}_{t} K f(\widehat{x})=\int(K f)(\widehat{y}) \widehat{p}_{t}(\widehat{x}, d \widehat{y}), \widehat{x} \in \widehat{X} \text { and } f \in \mathcal{B}^{b}(X)
$$

where $\widehat{p}_{t}(\widehat{x}, \widehat{E})=\widehat{P}_{t} 1_{\widehat{E}}(\widehat{x})$ is the probability transition function of $\widehat{M}$.

Remark 5: [18] Assumptions 1, 2 and 3 together imply the Rogers-Pitman intertwining relation [27], which are (10) and (11), (12), where

$$
\begin{aligned}
K \psi^{*} & =I, \\
\widehat{P}_{t} & =K P_{t} \psi^{*}
\end{aligned}
$$


Condition (11) says that the operator $K \psi^{*}$ is the identity of $\mathcal{B}^{b}(\widehat{X})$.

Remark 6: The equality (10) remains true if it is restricted to $\mathcal{B}^{b}(A)$. This results from Assumption 1 and Remark 4 .

Recall that the strong generator $\mathcal{L}$ of the process $M$ (for example) is the derivative of $P_{t}$ at $t=0$. Let $D(\mathcal{L}) \subset \mathcal{B}^{b}(X)$ be the set of functions $f$ for which the following limit exists (denoted by $\mathcal{L} f$ )

$$
\lim _{t \searrow 0} \frac{1}{t}\left(P_{t} f-f\right)
$$

The following proposition is a version of the Proposition 4.1 from [18], for the case when the processes involved are not necessarily Feller [4].

Proposition 1: Let $M, \widetilde{M}$ and $\widehat{M}$ have the respective generators $\mathcal{L}, \dot{\widetilde{\mathcal{L}}}$ and $\widehat{\mathcal{L}}$, that have domains, respectively, $D(\mathcal{L}), D(\widetilde{\mathcal{L}})$ and $D(\widehat{\mathcal{L}})$. Consider a vector space of functions $D \subset \mathcal{B}^{b}(\widetilde{X})$ such that the following conditions hold:

(i) $K \pi^{*}(D) \subset D(\widehat{\mathcal{L}})$ (hence $\psi^{*} K \pi^{*}(D) \subset D(\mathcal{L})$ );

(ii) $\phi^{*} K \pi^{*}(D) \subset D$;

(iii) there exists an appropriate extension $\mathcal{L}^{e}$ of the restriction of $\mathcal{L}$ to $\psi^{*} K \pi^{*}(D)$ with the domain $D\left(\mathcal{L}^{e}\right) \supseteq$ $\pi^{*}(D)$ such that $\mathcal{L}^{e}$ can be restricted to the vector space of measurable bounded $\mathbb{R}$-valued functions supported by $X \backslash A$ and it satisfies

$$
\begin{aligned}
K \mathcal{L}^{e} & =\widehat{\mathcal{L}} K \text { on } \pi^{*}(D) \\
\left(\mathcal{L}^{e} \pi^{*} \widetilde{f}\right)(x) & =\left(\mathcal{L}^{e} \pi^{*} \widetilde{f}\right)(y) \text { if } \widetilde{f} \in D \text { and } x \mathcal{R} y
\end{aligned}
$$

Then $D \subset D(\widetilde{\mathcal{L}})$ and

$$
\widetilde{\mathcal{L}} \widetilde{f}(\widetilde{x})= \begin{cases}\mathcal{L}^{e} \pi^{*} \widetilde{f}(\widetilde{x}) & \text { if } \widetilde{x} \in X \backslash A, \\ \widehat{\mathcal{L}} K \pi^{*} \widetilde{f}(\widetilde{x}) & \text { if } \widetilde{x} \in \psi(A) .\end{cases}
$$

Example 1: Consider the case when $M$ and $\widehat{M}$ are jump processes (or regular step processes in the terminology of [4]). Then

$$
\begin{aligned}
& (\mathcal{L} f)(x)=\lambda(x) \int_{X}[f(y)-f(x)] R(x, d y), x \in X \\
& (\widehat{\mathcal{L}} \widehat{f})(\widehat{x})=\widehat{\lambda}(\widehat{x}) \int_{\widehat{X}}[\widehat{f}(\widehat{y})-\widehat{f}(\widehat{x}) \widehat{R}(\widehat{x}, d \widehat{y}), \widehat{x} \in \widehat{X},
\end{aligned}
$$

where $\lambda$ and $\widehat{\lambda}$ are nonnegative and $R$ and $\widehat{R}$ are probability kernels. The Assumption 1 means that

$$
\begin{aligned}
\lambda & =\widehat{\lambda} \circ \psi \\
R\left(x, \psi^{-1}(\widehat{E})\right) & =\widehat{R}(\psi(x), \widehat{E}), x \in X \text { and } \widehat{E} \in \mathcal{B}(\widehat{X}) .
\end{aligned}
$$

The Assumption 3, when there exists a kernel $k$ which satisfies Assumption 2, is equivalent to

$$
\int_{X} R(y, E) k(\widehat{x}, d y)=\int_{\widehat{X}} k(\widehat{y}, E) \widehat{R}(\widehat{x}, d \widehat{y}),
$$

for $\widehat{x} \in \underset{X}{\widehat{X}}$ and $\widehat{E} \in \mathcal{B}(\widehat{X})$. Then, we define, for $\widetilde{x} \in \psi(A)$ and for $\widetilde{S} \in \mathcal{B}(\widetilde{X})$, the following probabilistic kernel

$$
\widetilde{R}(\widetilde{x}, \widetilde{S})=\widehat{R}(\widetilde{x}, \widetilde{S} \cap \psi(A))+\int_{\widehat{X} \backslash \psi(A)} k(\widehat{y}, \widetilde{S} \cap(X \backslash A)) \widehat{R}(\widetilde{x}, d \widehat{y}),
$$

and for $\widetilde{x} \in X \backslash A$ and for $\widetilde{S} \in \mathcal{B}(\widetilde{X})$,

$$
\begin{aligned}
\widetilde{R}(\widetilde{x}, \widetilde{S}) & =R(\widetilde{x}, \widetilde{S} \cap(X \backslash A))+\widehat{R}(\psi(\widetilde{x}), \widetilde{S} \cap \psi(A)) \\
& =R(\widetilde{x}, \widetilde{S} \cap(X \backslash A))+R\left(\widetilde{x}, \psi^{-1}(\widetilde{S}) \cap \psi(A)\right) .
\end{aligned}
$$

Then, we set the transition rate

$$
\widetilde{\lambda}(\widetilde{x})= \begin{cases}\lambda(\widetilde{x}) & \text { if } \widetilde{x} \in X \backslash A, \\ \widehat{\lambda}(\widetilde{x}) & \text { if } \widetilde{x} \in \psi(A) .\end{cases}
$$

In this case, the expression of $\widetilde{\mathcal{L}}$ is

$$
(\widetilde{\mathcal{L}} \widetilde{f})(\widetilde{x})=\widetilde{\lambda}(\widetilde{x}) \int_{\widetilde{X}}[\widetilde{f}(\widetilde{y})-\widetilde{f}(\widetilde{x})] \widetilde{R}(\widetilde{x}, d \widetilde{y}), \widetilde{x} \in \widetilde{X} .
$$

\section{VIEWPOINT INTEGRATION}

In this section we define an algorithm to construct the integration process from the viewpoint processes.

\section{A. The Constituents}

Let us consider the Borel right processes $M$ (with the semigroup $\left(P_{t}\right)$ ), $\widehat{M}_{i}$ (with the semigroup $\left(\widehat{P}_{t}^{i}\right)$ ), and the maps $\psi_{i}, i=1, . ., n$ as in the Subsection II-B.

Assumption 4: Assume that each $\psi_{i}, i=1, . ., n$ satisfies the Assumption 1, i.e. $\widehat{M}_{i}$ on $\psi_{i}\left(A_{i}\right)$ simulates $M$ on $A_{i}$. All the remarks from the Section II-C have shown that the methodology to construct new Markov processes, which exhibit a required behavior on a certain set, needs only

1) the local values of a surjective Markov function [27] on a corresponding set, and

2) some switching probabilistic kernels on that set. Let us define

$$
\widetilde{X}_{1}=\left(X \backslash A_{1}\right) \cup \psi_{1}\left(A_{1}\right)
$$

and $\pi_{1}: X \rightarrow \widetilde{X}_{1}$ given by

$$
\pi_{1}(x)= \begin{cases}x & \text { if } x \in X \backslash A_{1} \\ \psi_{1}(x) & \text { if } x \in A_{1} .\end{cases}
$$

Then, we define recursively, for $i=2, . ., n$ the spaces $\widetilde{X}_{i}=$ $\left(\widetilde{X}_{i-1} \backslash A_{i}\right) \cup \psi_{i}\left(A_{i}\right)$ and the projection maps $\pi_{i}: \widetilde{X}_{i-1} \rightarrow$ $\widetilde{X}_{i}$ given by

$$
\pi_{i}(x)= \begin{cases}x & \text { if } x \in \widetilde{X}_{i-1} \backslash A_{i} \\ \psi_{i}(x) & \text { if } x \in A_{i} .\end{cases}
$$

Let $\Pi_{i}=\pi_{i} \circ \pi_{i-1} \circ \ldots \circ \pi_{1}: X \rightarrow \widetilde{X}_{i}, i=1, . ., n$. It is clear that

$$
\Pi_{i}(x)= \begin{cases}x & \text { if } x \in X \backslash\left(A_{1} \cup \ldots \cup A_{i}\right) \\ \psi_{k}(x) & \text { if } x \in A_{k}, k=1, . ., i .\end{cases}
$$

The spaces $\widetilde{X}_{i}$ will be endowed with the topologies generated by the projection maps $\pi_{i}$. We assume that $\widetilde{X}_{i}$ with these topologies are Polish spaces. It is clear that

$$
\widetilde{X}=\widetilde{X}_{n}=\cup_{i=1}^{n} \psi_{i}\left(A_{i}\right)
$$

and $\Pi=\Pi_{n}: X \rightarrow \widetilde{X}, \Pi(x)=\psi_{i}(x)$ if $x \in A_{i}$ ( $\Pi$ does not depend on the composition order).

Our goal is to construct a new Markov process $\widetilde{\mathbf{M}}$ on $\widetilde{X}$ (the integration process), which behaves like $\widehat{M}_{i}$ on $\psi_{i}\left(A_{i}\right)$, $i=1, . ., n$. To complete the construction of $\widetilde{\mathbf{M}}$, we need to 
describe how $\widetilde{\mathbf{M}}$ passes from one location to another one. This is accomplished by some probabilistic kernels

$$
k_{i}: \widehat{X}_{i} \times \mathcal{B}(X) \rightarrow \mathbb{R}, i=1, . ., n
$$

that describe the jumping mechanism at the boundary of $\psi_{i}\left(A_{i}\right)$.

In order to be able to use the construction methodology presented in the Section II-C, we need to define the following auxiliary kernels

$$
\widetilde{k}_{1}=k_{1},
$$

and, for $i=2, . ., n$

$$
\begin{aligned}
\widetilde{k}_{i} & : \quad \widehat{X}_{i} \times \mathcal{B}\left(\widetilde{X}_{i-1}\right) \rightarrow \mathbb{R}, \\
\widetilde{k}_{i}(\widehat{x}, \widetilde{E}) & =k_{i}\left(\widehat{x}, \Pi_{i-1}^{-1}(\widetilde{E})\right),
\end{aligned}
$$

for all $\widehat{x} \in \widehat{X}_{i}, \widetilde{E} \in \mathcal{B}\left(\widetilde{X}_{i-1}\right)$.

Assumption 5: Assume that each $k_{i}, i=1, . ., n$ satisfies the Assumptions 2 and 3.

Any measurable set $\widetilde{E} \in \mathcal{B}\left(\widetilde{X}_{i-1}\right)$ can be decomposed as

$$
\widetilde{E}=\widetilde{F} \cup \widetilde{G},
$$

where

$$
\widetilde{F}=\widetilde{E} \backslash\left(A_{1} \cup \ldots \cup A_{i-1}\right)
$$

and

$$
\widetilde{G}=\widetilde{E} \cap\left(\psi_{1}\left(A_{1}\right) \cup \ldots \cup \psi_{i-1}\left(A_{i-1}\right)\right) .
$$

Then, we can obtain the following result about the auxiliary kernels $\widetilde{k}_{i}$.

Lemma 2: For any $\widetilde{E} \in \mathcal{B}\left(\widetilde{X}_{i-1}\right)$, the expression of $\widetilde{k}_{i}$, $i=2, . ., n$ is

$\widetilde{k}_{i}(\widehat{x}, \widetilde{E})= \begin{cases}k_{i}\left(\widehat{x}, \Pi_{i-1}^{-1}(\widetilde{G}) \cap \psi_{i}^{-1}(\widehat{x})\right), & \widehat{x} \in \widehat{X}_{i} \backslash \psi_{i}\left(A_{i}\right) \\ k_{i}\left(\widehat{x}, \widetilde{F} \cap \psi_{i}^{-1}(\widehat{x})\right), & \widehat{x} \in \psi_{i}\left(A_{i}\right)\end{cases}$

Proof: Using the Assumption 2, we obtain

$\widetilde{k}_{i}(\widehat{x}, \widetilde{E})=k_{i}\left(\widehat{x}, \Pi_{i-1}^{-1}(\widetilde{F})\right)+k_{i}\left(\widehat{x}, \Pi_{i-1}^{-1}(\widetilde{G})\right)$

$=k_{i}\left(\widehat{x}, \widetilde{F} \cap \psi_{i}^{-1}(\widehat{x})\right)+k_{i}\left(\widehat{x}, \Pi_{i-1}^{-1}(\widetilde{G}) \cap \psi_{i}^{-1}(\widehat{x})\right)$.

Then from the definition of $\Pi_{i-1}$ the result is clear. $\square$

Lemma 3: For all $i=2, . . n$ we have

$$
\begin{aligned}
\widetilde{K}_{i} & : \mathcal{B}^{b}\left(\widetilde{X}_{i-1}\right) \rightarrow \mathcal{B}^{b}\left(\widehat{X}_{i}\right) \\
\widetilde{K}_{i} \widetilde{f} & =K_{i}\left(\Pi_{i-1}^{*} \widetilde{f}\right)
\end{aligned}
$$

The algorithm to construct the desired process $\widetilde{M}$ consists in the iteration of the methodology presented in Section II-C. The Remarks 1, 3, 6 allow us to use at each step only the restrictions $\psi_{i}: A_{i} \rightarrow \psi_{i}\left(A_{i}\right)$ since the necessary Assumption 1 remains true. Moreover, from the same remarks we can obtain the following result.

Proposition 4: The restriction of the kernels $\widetilde{k}_{i}, i=$ $2, . ., n$ to

$$
\psi_{i}\left(A_{i}\right) \times \mathcal{B}\left(X \backslash\left(A_{1} \cup \ldots \cup A_{i-1}\right)\right)
$$

satisfy the Assumptions 2 and 3.

Proof: The result derives from Lemma 2 and from the fact that the kernels $k_{i}$ satisfy the required assumptions.
Therefore, the construction of the integration process will employ only the restrictions $\psi_{i}: A_{i} \rightarrow \psi_{i}\left(A_{i}\right)$ and the appropriate restrictions of the kernels $\widetilde{k}_{i}, i=1, . ., n$.

Informally, the construction of $\widetilde{\mathbf{M}}$ can be given as follows:

\section{Algorithm}

Set

$k=0, A_{k}=\emptyset$ and $Y_{k}=X \backslash A_{k}$.

\section{Repeat}

$k=k+1$

Choose $A_{k} \subset Y_{k-1}$ and the corresponding zigzag morphism $\psi_{k}$ restricted to $A_{k}$. $\left\{\right.$ It can be any $A_{i}, i=1, . ., n$ after re-indexing partition (2)\}

Construct a process $\widetilde{M}_{k}$, which behaves as $M$ on $Y_{k-1} \backslash A_{k}$, and as $\widehat{M}_{i}$ on $\psi_{i}\left(A_{i}\right), i=1, . . k$. Use the method presented in Section II-C. $\}$

Then $Y_{k}=Y_{k-1} \backslash A_{k}$.

\section{Until}

$Y_{k}=\emptyset$.

Proposition 5: The resulted process $\widetilde{\mathrm{M}}$ is a Borel right process with the state space $\widetilde{\mathbf{X}}=\cup_{i=1}^{n} \psi_{i}\left(A_{i}\right)$.

Proof: The methodology, presented in the Section II-C, ensures us that $\widetilde{\mathrm{M}}=\widetilde{M}_{n}$ is again a Borel right process. Moreover, at each step the expression of the generator for $\widetilde{M}_{k}$ can be written in terms of the generator of $M$ and $\widehat{M}_{i}$, $i=1, . . k$.

\section{B. The Generator}

One of the main mathematical results of this paper is related to the generator of the integration process $\mathbf{M}$. This generator can be used to characterize different functionals associated to the integration process. Moreover, it can be used to compute the transition probabilities of the integration process using the Kolmogorov backward equation (see [14], p. 30).

Theorem 6: Let $\widetilde{\mathbf{M}}$ and $\widehat{M}_{i}$ have the respective generators $\widetilde{\mathcal{L}}$ and $\widehat{\mathcal{L}}_{i}$, that have domains, respectively, $D(\widetilde{\mathcal{L}})$ and $D\left(\widehat{\mathcal{L}}_{i}\right)$, $i=1, . ., n$. If $\widetilde{f} \in \mathcal{B}^{b}(\widetilde{\mathbf{X}})$ then $\left.f_{i}=\left.\widetilde{f}\right|_{\psi_{i}\left(A_{i}\right.}\right) \in \mathcal{B}^{b}\left(\psi_{i}\left(A_{i}\right)\right)$ for, $i=1, . ., n$ and the expression of the generator is

$$
\widetilde{\mathcal{L}} \widetilde{f}(\widetilde{x})=\widehat{\mathcal{L}}_{i}\left(K_{i}\left(\widetilde{f}_{i} \circ \psi_{i}\right)\right)(\widetilde{x}), \widetilde{x} \in \psi_{i}\left(A_{i}\right)
$$

where $K_{i}$ and $\psi_{i}$ are appropriate restrictions, i.e.

$$
\begin{aligned}
K_{i} & : \quad \mathcal{B}^{b}\left(A_{i}\right) \rightarrow \mathcal{B}^{b}\left(\psi_{i}\left(A_{i}\right)\right) ; \\
\psi_{i} & : \quad A_{i} \rightarrow \psi_{i}\left(A_{i}\right)
\end{aligned}
$$

and $\widehat{\mathcal{L}}_{i}$ is understood as the generator of the restriction of $\widehat{M}_{i}$ to $\psi_{i}\left(A_{i}\right)$ (i.e. it is applied to the extension of $K_{i}\left(\widetilde{f}_{i} \circ \psi_{i}\right)$ with value 0 on $\widehat{X}_{i} \backslash \psi_{i}\left(A_{i}\right)$ ).

\section{The Case of Stochastic Hybrid Systems}

The applications of stochastic hybrid systems are ubiquitous. From traditional engineering domains as chemical engineering, control system, air traffic control, etc., the new applications include new fields like communication and computer networks [20], wireless communication gadgets. In particular, when applied to networks, stochastic hybrid systems can be thought as a class of fluid models. 
The stochastic hybrid systems can be thought as systems whose behaviours can be characterised as an interleaving of behaviours two stochastic systems: one discrete and another one continuous. Until now, we have considered only the situations when the stochastic viewpoints have the same nature, i.e. all are discrete or continuous. In a series of papers [13], [8] we have developed a model of stochastic hybrid systems whose behaviour is a strong Markov process as defined in Section 2. Consequently, the viewpoints that are defined as stochastic hybrid systems (in this model) can be integrated using the technique presented in this section. Remark that we know only the behaviour of the integration, but not the integrated system. At system level, one should consider a conformance relation between viewpoints, like in object $\mathrm{Z}$ where viewpoints are defined by conformant abstract data types. Roughly, a stochastic hybrid system comprises a (finite or countable) set of locations and discrete transitions between them. In each location, the system evolves as a random dynamical system [1]. The discrete transitions are governed by a Markov chain. Therefore, the integration should be defined for each component of stochastic hybrid system definition.

\section{FINAL REMARKS}

The systems, modelled the stochastic viewpoints introduced in this paper, can be both discrete or continuous. Traditionally, viewpoints have been considered for discrete (possibly infinite state) systems. The contribution of this paper is novel in considering both probabilistic and continuous system features. It is notorious the complexity of systems combining continuous behaviours and probabilities. Studying their interaction requires advanced use of functional and stochastic analysis. Always, it is a difficult choice to prioritise the possibilities of exploring the large modelling power by a great variety of examples and case studies or to build firm mathematical foundations of a complex paradigm. Our option is for soundness leaving illustrations and case studies for a following paper. It is expected that the sound method will encourage the development of its applications.

\section{REFERENCES}

[1] Arnold, L.: "Random Dynamical systems" Springer-Verlag, Berlin, (1998).

[2] Bemporad, A., Di Cairano, S.: Modelling and Optimal Control of Hybrid Systems with Event Uncertainty. In M. Morari and L. Thiele (Eds.), Proc. Hybrid Systems: Computation and Control, 8th International Workshop, LNCS 3414 (2005).

[3] Blom, H.A.P., Lygeros, J. (Eds.): "Stochastic Hybrid Systems: Theory and Safety Critical Applications". Springer Lecture Notes in Control and Information Sciences 337 (2006): 3-30.

[4] Blumenthal, R.M., Getoor, R.K.: "Markov Processes and Potential Theory". Academic Press, New York and London, (1968).

[5] Boiten E.A., Bujorianu M.C.: Exploring UML Refinement through Unification Workshop on Critical Systems Development with UML, $<<$ U M L $>>2003$, San Francisco, California, USA, October 20 24, 2003.

[6] Boiten E.A., J. Derrick, H. Bowman, M.W.A. Steen: Constructive consistency checking for partial specification in Z. Science of Computer Programming, 35(1), (1999): 29-75.

[7] Bravetti M., Hermanns H., Katoen J.-P.: YMCA -Why Markov Chain Algebra? in Proc. of PA'05: Algebraic Process Calculi: The First Twenty Five Years and Beyond, ENTCS, 2005.
[8] Bujorianu, M.L., Lygeros, J.: Towards Modelling of General Stochastic Hybrid Systems. In [3], pp. 3-30, (2006).

[9] Bujorianu, M.L., Bujorianu, M.C.: A Model Checking Strategy for a Class of Performance Properties of Fluid Stochastic Models. In M. Telek e.a. eds., Proceedings of 3rd European Performance Engineering Workshop, Springer LNCS, (2006) -In press.

[10] Bujorianu, M.L., Lygeros, J., Bujorianu, M.C.: Bisimulation for General Stochastic Hybrid Systems. In M. Morari and L. Thiele (Eds.), Proc. Hybrid Systems: Computation and Control, 8th International Workshop, LNCS 3414 (2005): 198-216.

[11] Bujorianu M.C., Boiten E.A.: Towards Correspondence Carrying Specifications, In C. Ratray, S. Maharaj, C. Shankland (eds.): "Algebraic Methodology and Software Technology. 10th International Conference AMAST 2004", Springer Verlag LNCS series, 2004.

[12] Bujorianu M.C.: Integration of Specification Languages Using Viewpoints. In E.A. Boiten, J. Derrick, G. Smith (eds.): "IFM 2004: Integrated Formal Methods" Canterbury, Kent, UK, Springer LNCS 2999, 2004..

[13] Bujorianu, M.L.: Extended Stochastic Hybrid Systems and their Reachability Problem. In R. Alur, G. Pappas (Eds.), Hybrid Systems: Computation and Control 7th International Workshop, LNCS 2993 (2004): 234-249.

[14] Davis, M.H.A.: "Markov Models and Optimization" Chapman \& Hall, London, (1993).

[15] C. Dutheillet, S. Haddad Regular Stochastic Petri Nets Advances in Petri Nets Springer LNCS 483 pp. 186-210, 1990

[16] Dynkin, E.B.: "Markov Processes". Vol.1. Berlin Heidelberg New York: Springer (1965).

[17] European Space Agency, Directorate Of Technical And Operational Support D / TOS / GSED / MSD / SIM XASTRO Data Exchange Framework Review DTOS-SST-TN-0689-TOS-GIC 2002 http://www.ccsds.org/docu/dscgi/ds.py/GetRepr/File-433/html

[18] Evans, S.N, Sowers, R.B.: Pinching and Twisting Markov Processes. The Annals of Probability 31 (1) (2003): 486-527.

[19] Finkelstein A., Kramer J., Nuseibeh B., Finkelstein L., Goedicke M.: Viewpoints: a framework for integrating multiple perspectives in system development. International Journal on Software Engineering and Knowledge Engineering, 2(1), pp. 31-58, 1992.

[20] Hespanha, J.P.: Stochastic Hybrid Systems: Application to Communication Network. In R. Alur, G. Pappas (Eds.), Hybrid Systems: Computation and Control 7th International Workshop, LNCS 2993 (2004): 387-401.

[21] IEEE Architecture Working Group IEEE P1471/D5.0 Information Technology -Draft Recommended Practice for Architectural Description, (1999).

[22] ITU Recommendation X.901-904 | ISO/IEC 10746 1-4. Open Distributed Processing - Reference Model - Parts 1-4, July 1995.

[23] Kang W., Kelly F. P., Lee, N. H., Williams, R. J.: Fluid and Brownian approximations for an Internet congestion control model. Proceedings of the 43rd IEEE Conference on Decision and Control (2004).

[24] Kelly F. P.: Mathematical modelling of the Internet. In B. Engquist, W. Schmid eds. "Mathematics Unlimited - 2001 and Beyond" SpringerVerlag (2001), 685-70.

[25] Kulkarni V., Trivedi K.S., FSPNs: Fluid Stochastic Petri Nets In Proc. 14th Int. Conf. On the Application and Theory of Petri Nets (1993) 24-31.

[26] NWO Project Description AiSHA: "Abstraction in Stochastic and Hybrid process Algebra" www.onderzoekinformatie.nl/en/oi/nod/onderzoek/OND1303139

[27] Rogers, L.C.G., Pitman, J.W.: Markov Functions. Ann. Prob., 9 (4), (1981): 573-582.

[28] Steen M., Derrick J., Boiten E.A., Bowman H.: Consistency of Partial Process Specifications. In A. Haeberer, ed. AMAST'98, Springer Verlag, 1999.

[29] Wolter K. Second Order Fluid Stochastic Petri Nets: an Extension of GSPNs for Approximate and Continuous Modeling In Proc. 1st World Congress on System Simulation (1997), 328-332. 